Louisiana State University

LSU Digital Commons

$1-1-2014$

\title{
Folate in demethylation: The crystal structure of the rat dimethylglycine dehydrogenase complexed with tetrahydrofolate
}

\author{
Zigmund Luka \\ Vanderbilt University Medical Center \\ Svetlana Pakhomova \\ Louisiana State University \\ Lioudmila V. Loukachevitch \\ Vanderbilt University Medical Center \\ Marcia E. Newcomer \\ Louisiana State University \\ Conrad Wagner \\ Vanderbilt University Medical Center
}

Follow this and additional works at: https://digitalcommons.Isu.edu/biosci_pubs

\section{Recommended Citation}

Luka, Z., Pakhomova, S., Loukachevitch, L., Newcomer, M., \& Wagner, C. (2014). Folate in demethylation: The crystal structure of the rat dimethylglycine dehydrogenase complexed with tetrahydrofolate. Biochemical and Biophysical Research Communications, 449 (4), 392-398. https://doi.org/10.1016/ j.bbrc.2014.05.064

This Article is brought to you for free and open access by the Department of Biological Sciences at LSU Digital Commons. It has been accepted for inclusion in Faculty Publications by an authorized administrator of LSU Digital Commons. For more information, please contact ir@lsu.edu. 


\title{
Folate in demethylation: the crystal structure of the rat dimethylglycine dehydrogenase complexed with tetrahydrofolate
}

\author{
Zigmund Luka ${ }^{1,}$, Svetlana Pakhomova ${ }^{2}$, Lioudmila V. Loukachevitch ${ }^{1}$, Marcia E. \\ Newcomer ${ }^{2}$, and Conrad Wagner ${ }^{1}$ \\ ${ }^{1}$ Department of Biochemistry, Vanderbilt University Medical Center, Nashville, Tennessee, 37232, \\ USA \\ 2Louisiana State University, Department of Biological Sciences, Baton Rouge, Louisiana, 70803, \\ USA
}

\begin{abstract}
Dimethylglycine dehydrogenase (DMGDH) is a mammalian mitochondrial enzyme which plays an important role in the utilization of methyl groups derived from choline. DMGDH is a flavin containing enzyme which catalyzes the oxidative demethylation of dimethylglycine in vitro with the formation of sarcosine (N-methylglycine), hydrogen peroxide and formaldehyde. DMGDH binds tetrahydrofolate (THF) in vivo, which serves as an acceptor of formaldehyde and in the cell the product of the reaction is 5,10-methylene tetrahydrofolate instead of formaldehyde. To gain insight into the mechanism of the reaction we solved the crystal structures of the recombinant mature and precursor forms of rat DMGDH and DMGDH-THF complexes. Both forms of DMGDH reveal similar kinetic parameters and have the same tertiary structure fold with two domains formed by $\mathrm{N}$ - and $\mathrm{C}$-terminal halves of the protein. The active center is located in the $\mathrm{N}$ terminal domain while the THF binding site is located in the C-terminal domain about $40 \AA$ from the isoalloxazine ring of FAD. The folate binding site is connected with the enzyme active center via an intramolecular channel. This suggests the possible transfer of the intermediate imine of dimethylglycine from the active center to the bound THF where they could react producing a 5,10methylenetetrahydrofolate. Based on the homology of the rat and human DMGDH the structural basis for the mechanism of inactivation of the human DMGDH by naturally occurring His109Arg mutation is proposed
\end{abstract}

\footnotetext{
(C) 2014 Elsevier Inc. All rights reserved.

*To whom correspondence should be addressed: Zigmund Luka, 604 Robinson Research Building, Vanderbilt University Medical Center, Nashville, TN 37232. Tel:1-615-343-6572; Fax: 1-615-343-0704; z.luka@ vanderbilt.edu.

Supplementary data.

Supplementary data associated with this article can be found in the online version.

Publisher's Disclaimer: This is a PDF file of an unedited manuscript that has been accepted for publication. As a service to our customers we are providing this early version of the manuscript. The manuscript will undergo copyediting, typesetting, and review of the resulting proof before it is published in its final citable form. Please note that during the production process errors may be discovered which could affect the content, and all legal disclaimers that apply to the journal pertain.
} 


\section{Keywords}

dimethylglycine dehydrogenase; rat; tetrahydrofolate; crystal structure

\section{Introduction}

DMGDH is a mammalian flavin mitochondrial enzyme (EC 1.5.8.4), which catalyzes the oxidative demethylation of dimethylglycine (DMG). When this reaction is studied in vitro the products of reaction are sarcosine, hydrogen peroxide and formaldehyde [1, 2]. In the cell, the redox partner of DMGDH is an electron-transferring flavoprotein (ETF) [3]. The demethylation is performed using FAD as electron acceptor which is covalently bound to His84 (precursor numbering) via a histidyl(N3)-(8a)FAD bond [4]. In the rat this enzyme is encoded by a nuclear gene and is translated as a precursor protein of 857-aa (molecular mass of $96 \mathrm{kDa}$ ) [5]. Upon transfer into mitochondria the 21-aa N-terminal mitochondrial targeting peptide is removed by a mitochondrial processing protease [6].

The physiological role of both the substrate and product of the DMGDH reaction, dimethylglycine and sarcosine, are not well understood although they are important intermediates in one-carbon metabolism. Abnormal levels of DMG are associated with a variety of diseases and DMG has been proposed as therapeutic for specific pathologies [79].

This enzyme was re-discovered in our laboratory as a folate-binding protein [10]. It binds different forms of folate with the highest affinity for THF. Based on that finding it was proposed that bound THF serves as a scavenger for the formaldehyde produced during the course of dimethylglycine demethylation. The mechanism of this process could not be determined because of the lack of structural data.

We have now solved the crystal structures of the mature and precursor forms of the rat DMGDH in the presence of THF. Our choice of the rat DMGDH was based on the fact that this enzyme was much better studied than any other mammalian DMGDH, thus structure/ function relationships can be precisely described.

\section{Materials and Methods}

A recombinant precursor (prDMGDH) and mature (mDMGDH) forms of rat DMGDH cloned from Mammalian Gene Collection clone (RefSeq BC085697) were expressed in E. coli. In addition, precursor form of rat DMGDH (prDMGDH-var2) was expressed from the plasmid provided by Dr. Barile (University of Bari, Italy). In the latter there are six substitutions in amino acids as explained below. Cloning, proteins expression and purification are described in Supplementary Data.

\section{Activity Assay}

DMGDH steady state kinetics was studied by using ferricenium hexafluorophosphate $\left(\mathrm{Fc}^{+} \mathrm{PF}_{6}^{-}\right)$[11]. This synthetic one-electron acceptor changes its spectrum upon reduction to ferrocene by $\mathrm{FADH}_{2}$. An assay was carried out in a $150 \mu \mathrm{l}$ reaction mixture containing 100 
mM HEPES pH 7.5, $35 \mathrm{nM}$ of enzyme, $200 \mu \mathrm{M}$ of $\mathrm{Fc}^{+} \mathrm{PF}_{6}{ }^{-}$and $0.05-50 \mathrm{mM}$ of N,Ndimethylglycine at $25^{\circ} \mathrm{C}$. The reduction of $\mathrm{Fc}^{+} \mathrm{PF}_{6}{ }^{-}$was followed by the decrease of absorbance at $300 \mathrm{~nm}$ using a Shimadzu $2540 \mathrm{UV} /$ visible spectrophotometer equipped with a thermostated cell holder. The DMGDH activity was calculated by moles of reduced $\mathrm{Fc}^{+} \mathrm{PF}_{6}{ }^{-}$which was calculated based on $\varepsilon_{300}=4300 \mathrm{M}^{-1} \mathrm{~cm}^{-1}$ [11] taking into account that 2 moles of $\mathrm{Fc}^{+} \mathrm{PF}_{6}{ }^{-}$are reduced per mole of enzyme. Measurements were made in duplicate. Kinetic data were analyzed by the Michaelis-Menten equation using GraphPad Prism 5 software.

\section{Crystallization}

The mature form of DMGDH was crystallized using $0.2 \mathrm{M}$ K/Na-tartrate-20\% PEG 3350 as a precipitant. The protein solution in $20 \mathrm{mM}$ HEPES pH 7.5-25 mM NaCl- $2 \mathrm{mM}$ DTT with protein concentration of $12-15 \mathrm{mg} / \mathrm{ml}$ was mixed with precipitant.

The precursor DMGDHvar-2 was crystallized using 0.2 M NaSCN-20\% PEG 3350-0.1 M Tris $\mathrm{pH} 7.5$ as precipitant. The protein solution in $20 \mathrm{mM}$ HEPES $\mathrm{pH} 7.5-25 \mathrm{mM} \mathrm{NaCl}-2$ $\mathrm{mM}$ DTT with a protein concentration of $12-15 \mathrm{mg} / \mathrm{ml}$ was mixed with precipitant at $20^{\circ} \mathrm{C}$.

Folate was introduced into the crystals by the soaking method in the precipitant solutions used for crystallization supplemented with $25 \mathrm{mM}$ DTT and 5-10 mM THF for three hours at $20^{\circ} \mathrm{C}$. After soaking crystals were flash-frozen in liquid nitrogen and used for the X-Ray data collection. THF concentration was determined by absorbance at the maximum at 298 $\mathrm{nm}$ with an extinction coefficient at $\mathrm{pH} 7.0$ of $29.1 \mathrm{mM}^{-1} \mathrm{~cm}^{-1}$.

\section{Data collection and structure solving}

Diffraction data were collected at $100 \mathrm{~K}$ at LS-CAT beamline 21-F, Advanced Photon Source, Argonne National Laboratory using Mar225 CCD detector. Data were processed and scaled using HKL2000 package [12]. Data collection and data processing statistics are summarized in Table 1.

\section{Crystal structure determination}

The first and superior data set was collected for the precursor DMGDHvar-2-folate crystal and the crystal structure was determined for that protein. The structure was solved by Molecular Replacement (MR) with MOLREP [13]. A monomer of bacterial dimethylglycine oxidase (PDB accession code 1PJ5) served as the search model. Two protein molecules were located in the asymmetric unit. The positioned MR model was refined using the maximum likelihood refinement in REFMAC [13] with the TLS parameters generated by the TLSMD server [14]. Coot [15] was used for model building throughout the refinement. The final model consists of protein residues 38-860 (monomer A), 38-861 (monomer B), two covalently bound FAD molecules, two folate molecules, 8 thiocyanate anions and 721 water molecules. Alternate conformations have been built for protein residues K100, N693 (monomer A) and K100 (monomer B).

The refined DMGDH-var2-folate structure at $1.85 \AA$ resolution (with deletion of folate and water molecules from the model) was used to locate MR solutions in the mDMGDH and 
mDMGDH-folate data sets. Model building and refinement of these structures were done in a similar way as described for the prDMGDHvar-2-folate structure. Data refinement statistics are listed in Table 1. The data were deposited to Protein Data Bank with PDB IDs 4P9S, 4PAA and 4PAB for mature DMGDH, mature DMGDH-THF and precursor DMGDH-THF structures respectively.

\section{Results}

\section{Protein expression and activity}

The expression and purification protocol we used resulted in preparation of DMGDH precursor and mature forms completely reconstituted with FAD. Reconstitution efficiency was determined by the ratio of A275/A460. This parameter for native DMGDH prepared from rat liver mitochondria is 1.73 [16]. Our preparations routinely have absorbance ratios of $1.71-1.75$.

The main kinetic parameters are similar for both forms of DMGDH: there are only modest differences among the forms assayed. The precursor form is a slower enzyme with $\mathrm{k}_{\text {cat }}$ value (1.43-1.48 sec-1$)$, two fold lower compared to the mature form $\left(2.65 \mathrm{sec}^{-1}\right)$. Interestingly, the $\mathrm{K}_{\mathrm{m}}$ value for mature form $(580 \mathrm{mM})$ is lower than precursor DMGDH $(760 \mathrm{mM})$ but higher than prDMGDH-var2 $(460 \mathrm{mM})$. In the dimethylglycine concentrations used (1-50 $\mathrm{mM}$ ) our samples were characterized by single $\mathrm{K}_{\mathrm{m}}$ values as shown by transformation of standard velocity/substrate dependence into an Eadie-Hofstee plot (Supplementary figure 1 and Table 2).

\section{Overall structure}

A search of structures deposited in databank reveals only the bacterial DMGO [17-19] as a protein with structural similarity to full length DMGDH (Supplemental figure 2). RMS deviations over 742 structurally equivalent CA atoms (PDB code $3 \mathrm{GSI}$ ) are $1.88 \AA$. As in DMGO, DMGDH consists of two principle domains: the FAD binding domain (N-terminus domain, residues 1-450) and the folate binding domain (C-terminus domain, 451-860) as shown in Fig. 1A. The six amino acid residues in which correct and variant-2 sequences are different (Ala204Val, Thr402Lys, Asn746Asp, Asn774Asp, Ile267Val and Glu509Arg) are not likely to interfere with folding. RMS deviation between all three DMGDH structures described here lie in the $0.14-0.35 \AA$ range. The structures of precursor and mature forms of DMGDH are equivalent, the discussion reflects this fact.

\section{$\mathrm{N}$-terminus domain}

The N-terminal domain is 31 amino acids longer in DMGDH in comparison to the DMGO molecule. The residues 1-37 are not visible in electron density, consistent with a lack of order. Alignment of the N-terminal domain using the secondary structure matching (SSM) database [20] showed that the most structurally related protein is the $\beta$-subunit of L-proline dehydrogenase (29\% of sequence identity, RMSD of $1.65 \AA$ over the 349 CA atoms). Other matches include the $\beta$-subunit of sarcosine oxidase (24\% of sequence identity, RMSD of $1.97 \AA$ over the 349 CA atoms), MTOX (18\% of sequence identity, RMSD $2.26 \AA$ over 342 CA atoms), glycine oxidase ( $23 \%$ of sequence identity, RMSD of $2.31 \AA ̊$ over 334 CA 
atoms), D-arginine dehydrogenase (20\% of sequence identity, RMSD of $2.62 \AA$ over the 335 CA atoms) and nikD (18\% of sequence identity, RMSD of $2.72 \AA$ over $333 \mathrm{CA}$ atoms). The $\mathrm{N}$-terminus domain could be divided to two subdomains (Fig. 1B). The first one, (FADbinding subdomain) (residues 1-86, 188-258 and 368-450) is represented by a central fivestranded parallel $\beta$-sheet surrounded by a-helices on one side and three-stranded antiparallel $\beta$-sheet on the other side. Such nucleotide-binding fold is observed in enzymes belonging to the glutathione reductase family of enzymes. $\mathrm{N}$-terminal domain covalently binds cofactor FAD through His84.

The second domain, often called as "catalytic" domain in proteins with similar fold (residues 87-187 and 259-367), consists of a mixed, mostly antiparallel eight $\beta$-sheet surrounded by four a-helices. This domain wraps around the catalytic site.

The FAD molecule adopts an elongated conformation with the adenine and isoalloxazine moieties distal from each other, similar to the conformation observed in a number of other flavoproteins. The flavin isoalloxazine ring binds in the crevice between the two subdomains and is buried inside of the protein. In contrast, the adenine moiety is exposed to the solvent. FAD binding is stabilized by numerous hydrogen bonds in addition to the covalent linkage through histidyl(N3)-(8a)FAD (Fig. 1C). The N-3 of the flavin isoalloxazine ring makes hydrogen bond to the carbonyl oxygen of Ile $88, \mathrm{O}-4$ is hydrogen bonded to the main chain $\mathrm{NH}$ of Ile88, O-2 makes H-bonds to the main chain NHs of Ile394 and Ile395 and N-5 is hydrogen bonded to the main chain NH of Ala86. The ribityl phosphate moiety is stabilized through hydrogen bonding interactions between $\mathrm{O}^{\prime}$ and $\mathrm{OG}$ of Thr82, $\mathrm{O}^{\prime}$ and the carbonyl oxygen of Phe390 and the main chain NH of Gly393. Hydrophobic interactions with protein involve Tyr392, Ile365, Tyr265, Ile394, His263 and Ala85. The adenine ring sits in the hydrophobic pocket formed by Val212, Ile48, Trp244 and Ala241. Interactions of the adenine ring with the protein involve hydrogen bonds between $\mathrm{N}-1$ and the main chain $\mathrm{NH}$ of Val212, between N-6 and the carbonyl oxygen of Val212 and between N-3 and the main chain $\mathrm{NH}$ of Lys74. Additional interactions between FAD and protein include $\mathrm{H}$-bonds between $\mathrm{O} 2 \mathrm{~B}$ of the ribose ring and $\mathrm{O} 2 \mathrm{E}$ of Glu73, O3B of the ribose ring and $\mathrm{O} 1 \mathrm{E}$ of Glu73, O1A and the main chain NH of Ser81, O2A and the main chain NH and OG of Thr82.

\section{Folate binding domain}

Alignment of the folate binding domain showed that the same fold is observed in the Tprotein of the glycine cleavage system (30\% of sequence identity, RMSD of $1.35 \AA$ over $352 \mathrm{CA}$ atoms), aminomethyltransferase (31\% of sequence identity, RMSD of $1.65 \AA$ A over $349 \mathrm{CA}$ atoms) and dimethylsulfoniopropionate-dependent demethylase (19\% of sequence identity, RMSD of $1.78 \AA$ Aver $355 \mathrm{CA}$ atoms). The folate binding domain of DMGDH can be divided into three subdomains positioned in a cloverleaf-like arrangement as in the DMGO crystal structure (Fig. 2A). Domain 1 (residues 460-530 and 620-721) contains a single Greek Key motif surrounded by $\alpha$-helices. Domain 2 (residues 531-619 and 722763 ) is represented by five-stranded antiparallel $\beta$-sheet with flanking $\alpha$-helices. Domain 3 (residues 764-843) forms a distorted jelly roll. The striking feature of the DMGDH 
molecule in comparison to known structures of similar fold is a presence of an additional ahelix at the very end of the C-terminus, protruding into external solvent area.

The folate molecule is bound in the central cavity of the folate-binding domain (Fig. 2B) with the pterin ring located in the center of the cavity with the glutamate tail exposed to the external solvent. The bound folate is found in the bent conformation as observed in the DMGO-folate complex $[18,19]$. Surprisingly, binding of folate does not induce any conformational changes in the protein molecule. There are basically no differences in the conformation of amino acids lining the folate binding site between the folate bound and folate free forms of DMGDH. The RMSDs between the two structures lie in the 0.14-0.28 $\AA$ range for two independent molecules. The $\mathrm{N}-2$ of the pterin ring of folate makes hydrogen bonds with OH of Tyr678 and OE1 of Glu676 (Fig. 2C). N-3 is hydrogen bonded to OE2 of Glu676, N-1 to OE1 of Glu573. There is only one H-bond between the glutamate tail of folate: $\mathrm{OE} 2$ to $\mathrm{OH}$ of Tyr737. However, the water mediated hydrogen bond to the "corkscrew" helix OG atom of Thr843 is also observed. Additional stabilizing interactions include hydrophobic interactions with Ile667, Tyr669, Ile587, Thr575, Ile560, Phe738, Phe649, Leu650 and Phe717.

\section{The "corkscrew" $a$-helix}

The crystals which structures described in this work are crystallized in different crystal forms (Table 1). However, the "corkscrew" $a$-helix is presented in all crystal forms despite completely different packing contacts involving the helix. This fact indicates that formation of the terminal helix is not a packing artifact. The role of the "corkscrew" helix in DMGDH is unclear. Such a helix does not exist in DMGO and all C-terminus structurally related proteins, indicating that it is most likely not essential for the catalysis. However, the "corkscrew" $a$-helix (Fig. 2B) is positioned next to the folate binding site and there is a water mediated H-bond between OX1 of glutamate tail of folate and OG of Thr843. More work must be done to uncover the potential role of the "corkscrew" helix.

\section{Channel connection of the active center and the folate binding site}

The identical reactions catalyzed by rat DMGDH and DMGO from Arthrobacter globiformis and the similarity of the protein folds of these enzymes suggest that it is most likely the mechanism of the transfer of the one carbon unit to THF is the same or similar for both enzymes. For DMGO it was suggested that the intermediate imine from the active center is channeled to the folate binding site by an intramolecular channel [18, 19]. Analysis of the crystal structure of DMGDH revealed, indeed, that such a channel filled with numerous water molecules does exist in the mammalian enzyme. As shown in Fig. 3 a channel of about $40 \AA$, connects the enzyme active center with bound FAD and the folate binding site.

\section{Discussion}

The purpose of this work was to obtain a structural basis for the role of THF in the dimethylglycine demethylation by rat DMGDH since no structure of mammalian enzymes was solved so far. The DMG demethylation enzymes are important in one-carbon 
metabolism in mammals and prokaryotes. The common feature of the enzymes is that they bind THF, a scavenger for the otherwise toxic formaldehyde as a product of this reaction. Previously, the crystal structure of dimethylglycine oxidase from the bacterium Arthrobacter globiformis, with different forms of folates, was obtained [18, 19]. It was striking to discover that the folate binding site was localized about $40 \AA$ from the enzyme active center in the crystal structure of DMGO. Careful analysis of the structure of DMGO allowed the authors $[18,19]$ to propose the hypothesis that in the reaction catalyzed by DMGO, formaldehyde was not formed. They proposed that an intermediate imine formed from dimethylglycine is channeled from the active center to the THF forming 5,10methyleneTHF and released as a product of the reaction.

Analysis of the structure of rat DMGDH shows that the mechanism of the channeling of an intermediate imine proposed for DMGO could be applied to DMGDH. A possible channel from FAD to THF is shown in Fig. 3. We should notice here, that the channeling hypothesis still needs direct evidence for the existence of intermediates of the reaction of an imine with THF. This could be a very challenging project due to a potentially very short time of the existence of such intermediates as was pointed out elsewhere [19].

The crystal structure of rat DMGDH presented here is a good representation of mammalian DMGDH due to high similarity of the protein sequences. The human DMGDH precursor sequence is $91 \%$ identical to rat enzyme with one third of the differences located in the first $60 \mathrm{~N}$-terminal parts of the proteins. Therefore the crystal structure of rat DMGDH can be used in the explanation of the inactivation of the natural His109Arg mutant of human DMGDH [21]. The authors modeled the mechanism for that inactivation based on the crystal structure of DMGO from Arthrobacter globiformis. In their model the His 109 is located about $18 \AA$ from the FAD and therefore there is no direct participation in the formation of active center. Having the rat DMGDH structure solved and almost 100\% similarity with human enzyme in the region of interest we can now propose a more specific explanation of the effect of His109Arg mutation. The His109 in human DMGDH corresponds to His102 in the rat enzyme. In rat DMGDH this residue is located on the side of the helix 101-114 and is positioned toward FAD at a distance of $7.7 \AA$ (Fig. 4). Most importantly, His 102 establishes strong hydrogen bonds with Thr90 and His396 which means that it participates in creating the active center of the enzyme. This may indicate a strong interference in the conformation of the active center by replacements of histidine by arginine in the patient mutated enzyme and, as the result, a loss of activity.

In conclusion, the crystal structure we reported in this work is a necessary basis for the future studies of the mechanism of participation of the THF in dimethylglycine dehydrogenase enzymatic demethylation of dimethylglycine as well as in the other similar enzymes. It should be noted here that in our recently solved structure of lysine specific histone demethylase LSD1 complexed with tetrahydrofolate [22] the latter is bound in close proximity to FAD, thus providing another example of the importance of the study of the role of folate in demethylation. 


\section{Supplementary Material}

Refer to Web version on PubMed Central for supplementary material.

\section{Acknowledgments}

The authors thank EPROVA (Switzerland) for free samples of folate and Dr. Barile (University of Bari, Italy) for expression vector of rat DMGDH.

Thus study was supported from NIH grant DK15289 to C. W. and from Louisiana Governor's Biotechnology Initiative to M. E. N. The authors thank Vanderbilt University Center for Structural Biology for access to Biomolecular Crystallography Facilities.

Use of the Advanced Photon Source, an Office of Science User Facility operated for the U.S. Department of Energy (DOE) Office of Science by Argonne National Laboratory, was supported by the U.S. DOE under Contract No. DE-AC02-06CH11357. Use of the LS-CAT Sector 21 was supported by the Michigan Economic Development Corporation and the Michigan Technology Tri-Corridor (Grant 085P1000817).

\section{Abbreviations}

DMGDH dimethylglycine dehydrogenase

DMG dimethylglycine

THF tetrahydrofolate

\section{References}

1. Mackenzie CG, Frisell WR. The metabolism of dimethylglycine by liver mitochondria. J Biol Chem. 1958; 232:417-427. [PubMed: 13549430]

2. Porter DH, Cook RJ, Wagner C. Enzymatic properties of dimethylglycine dehydrogenase and sarcosine dehydrogenase from rat liver. Arch Biochem Biophys. 1985; 243:396-407. [PubMed: 2417560]

3. Hoskins DD, Mackenzie CG. Solubilization and electron transfer flavoprtein requirement of mitochondrial sarcosine dehydrogenase and dimethylglycine dehydrogenase. J Biol Chem. 1961; 236:177-83. [PubMed: 13716069]

4. Cook RJ, Misono KS, Wagner C. The amino acid sequences of the flavin-peptides of dimethylglycine dehydrogenase and sarcosine dehydrogenase from rat liver mitochondria. J Biol Chem. 1985; 260:12998-13002. [PubMed: 4055729]

5. Lang H, Polster M, Brandsch R. Rat liver dimethylglycine dehydrogenase. Flavinylation of the enzyme in hepatocytes in primary culture and characterization of a cDNA clone. Eur J Biochem. 1991; 198:793-799. [PubMed: 1710985]

6. Stoltz M, Rysavy P, Kalousek F, Brandsch R. Folding, flavinylation, and mitochondrial import of 6hydroxy-D-nicotine oxidase fused to the presequence of rat dimethylglycine dehydrogenase. J Biol Chem. 1995; 270:8016-8022. [PubMed: 7713902]

7. Freed WJ. Prevention of strychnine-induced seizures and death by the N-methylated glycine derivative betaine, dimethylglycine and sarcosine. Pharmacology, Biochemistry and Behavior. 1985; 22:641-643.

8. Kern JK, Miller VS, Cauller PL, et al. Effectiveness of N,N-dimethylglycine in autism and pervasive developmental disorder. J Child Neurol. 2001; 16:169-173. [PubMed: 11305684]

9. Svingen GF, Ueland PM, Pedersen EK, et al. Plasma dimethylglycine and risk of incident acute myocardial infarction in patients with stable angina pectoris. Arterioscler Thromb Vasc Biol. 2013; 33:2041-8. [PubMed: 23723367] 
10. Wittwer AJ, Wagner C. Identification of the folate-binding proteins of rat liver mitochondria as dimethylglycine dehydrogenase and sarcosine dehydrogenase. Flavoprotein nature and enzymatic properties of the purified proteins. J Biol Chem. 1981; 256:4109-4115. [PubMed: 6163778]

11. Lehman TC, Thorpe C. Alternate electron acceptors for medium chain acyl-CoA dehydrogenase: Use of ferricenium salts. Biochemistry. 1990; 29:10594-10602. [PubMed: 2271671]

12. Otwinowski Z, Minor W. Processing of X-ray diffraction data collected in oscillation mode. Methods in Enzymology. 1997; 276Macromolecular Crystallography, part A. :307-326.

13. Bailey S. The Ccp4 suite - programs for protein crystallography. Acta Crystallogr D-Biological Crystallography. 1994; 50:760-763.

14. Painter J, Merritt EA. TLSMD web server for the generation of multi-group TLS models. J Appl Crystallogr. 2006; 39:109-111.

15. Emsley P, Lohkamp B, Scott W, Cowtan K. Features and development of Coot. Acta Crystallographica Section D - Biological Crystallography. 2010; 66:486-501.

16. Cook RJ, Wagner C. Dimethylglycine dehydrogenase and sarcosine dehydrogenase: mitochondrial folate-binding proteins from rat liver. Methods Enzymol. 1986; 122:255-60. [PubMed: 3702694]

17. Meskys R, Harris RJ, Casaite V, et al. Organization of the genes involved in dimethylglycine and sarcosine degradation in Arthrobacter spp.: implications for glycine betaine catabolism. Eur J Biochem. 2001; 268:3390-3398. [PubMed: 11422368]

18. Leys D, Basran J, Scrutton NS. Channelling and formation of 'active' formaldehyde in dimethylglycine oxidase. EMBO J. 2003; 22:4038-4048. [PubMed: 12912903]

19. Tralau T, Lafite P, Levy C, et al. An internal reaction chamber in dimethylglycine oxidase provides efficient protection from exposure to toxic formaldehyde. J Biol Chem. 2009; 284:17826-17834. [PubMed: 19369258]

20. Krissinel E, Henrick K. Secondary-structure matching (SSM), a new tool for fast protein structure alignment in three dimensions. Acta Crystallogr D Biol Crystallogr. 2004; 60:2256-2268. [PubMed: 15572779]

21. McAndrew RP, Vockley J, Kim JJ, et al. Molecular basis of dimethylglycine Dehydrogenase deficiency associated with pathogenic variant H109R. J Inherit Metab Dis. 2008; 31:761-768. [PubMed: 18937046]

22. Luka Z, Pakhomova S, Loukachevitch LV, et al. Crystal structure of the histone lysine specific demethylase LSD1 complexed with tetrahydrofolate. Protein Sci. 2014 Epub ahead of print. 10.1002/pro.2469 


\section{Highlights}

- DMGDH is an important enzyme in one-carbon metabolism

- Bound tetrahydrofolate (THF) serves as a scavenger for the formaldehyde product

- We solved the crystal structure of DMGDH and DMGDH-THF complex

- An intramolecular channel connects the active site and the THF binding site

- A mechanism for human DMGDH inactivation by a natural mutation is proposed 


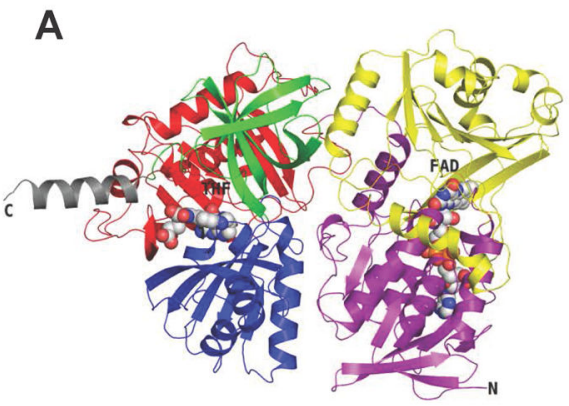

B

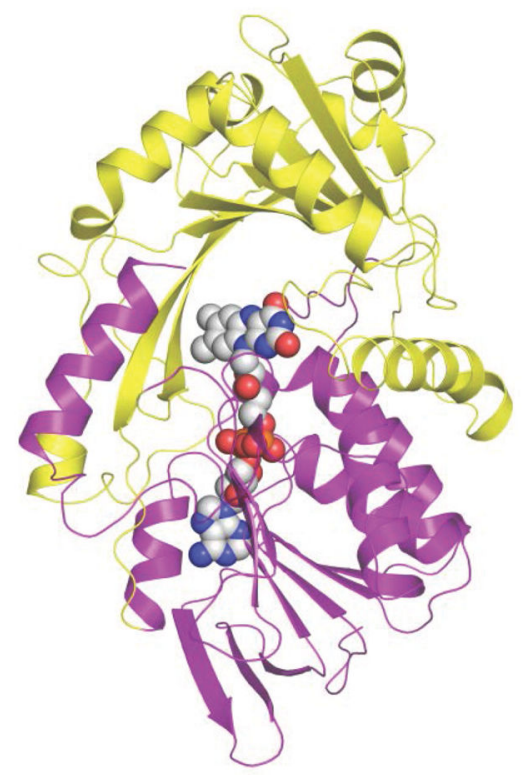

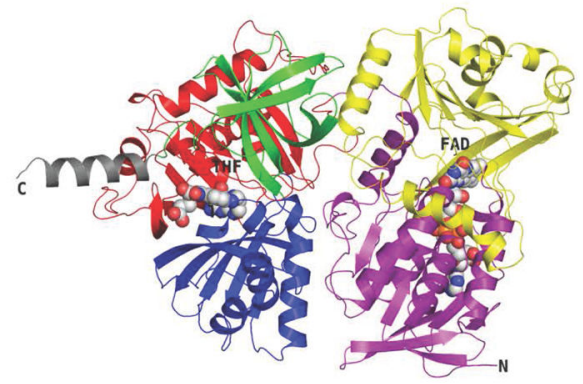

C

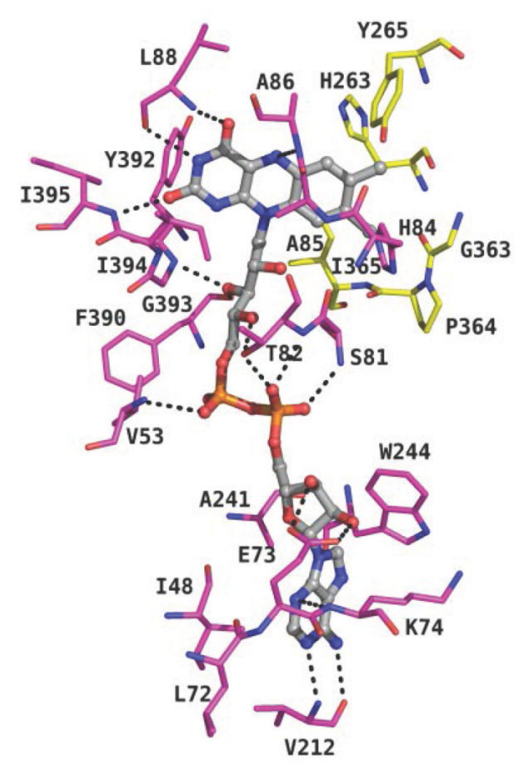

Figure 1. A. Stereo view of a monomer of DMGDH

The N-terminus FAD-binding domain (purple and yellow) and the C-terminus folatebinding domain (red, blue and green) are shown with secondary structure drawing. FAD and THF molecules are shown as space-filled models.

B. Structure of the N-terminus domain. The FAD binding subdomain is shown in purple, the "catalytic" subdomain is shown in yellow. FAD molecule is shown as space-filled model.

C. The FAD binding site. The amino acids from the FAD binding subdomain are shown in purple while the amino acids from the catalytic subdomain are shown in yellow. Dashed lines indicate hydrogen bonds. FAD molecule is shown in balls and sticks representation. 
A
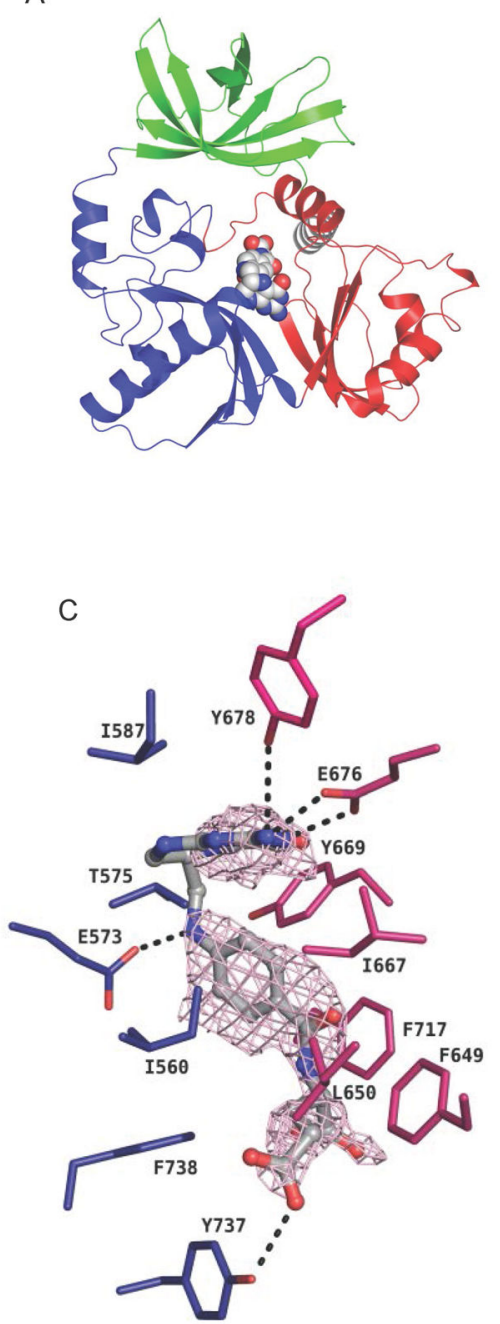

B
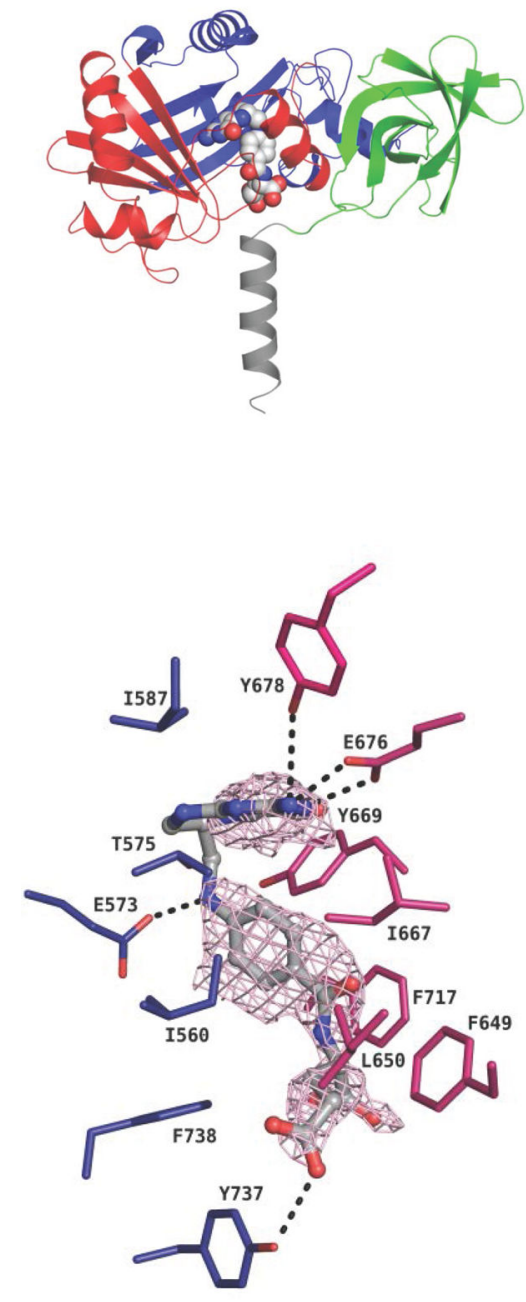

Figure 2. Structure of the folate-binding domain

A. View of domain in two orientations. The subdomain-1 is shown in red, the subdomain-2 is shown in blue and the subdomain-3 is shown in green. The terminal "corkscrew" a-helix is shown in grey. Folate molecule is shown as a space-filled model.

B. The stereo view of the folate binding site. The amino acids from the subdomain 1 are shown in red while the amino acids from the subdomain 2 are shown in blue. Dashed lines indicate hydrogen bonds. Fo-Fc electron density omit map contoured at $3 \sigma$. The folate molecule is shown in ball and stick representation. 


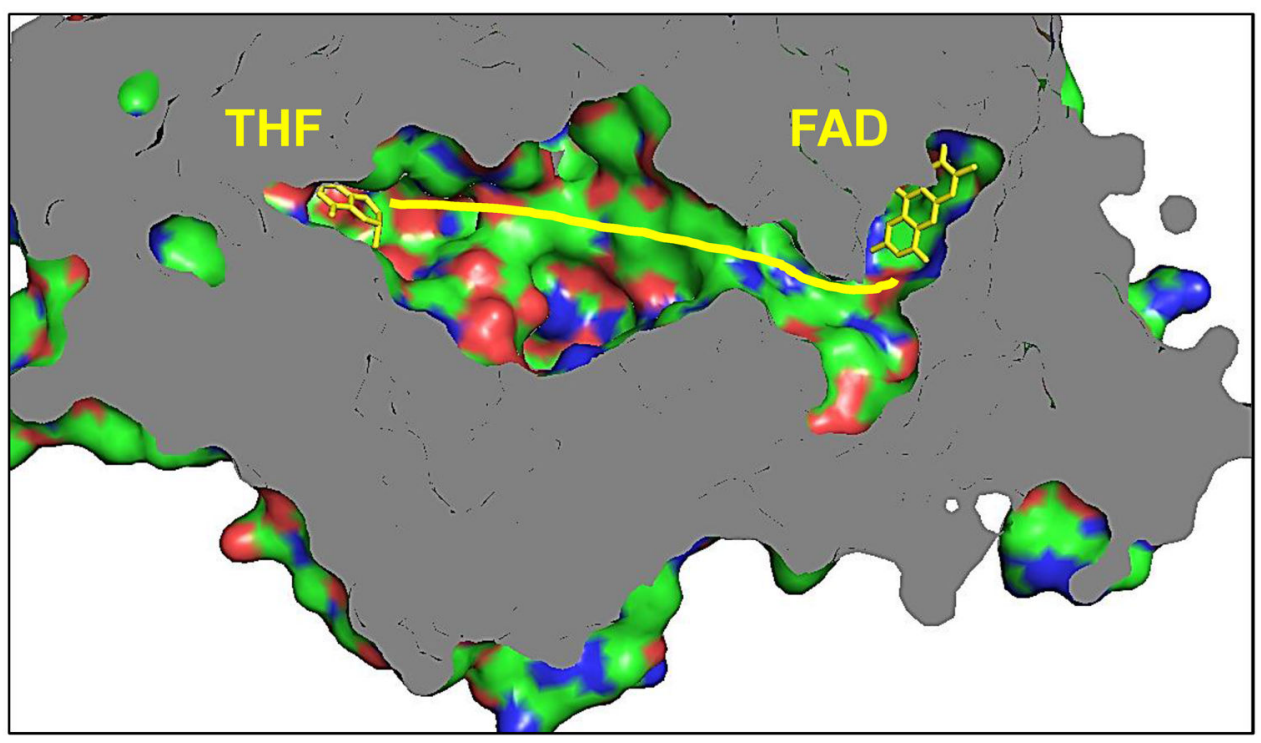

Figure 3. Intramolecular channel in DMGDH

The crossection of the DMGDH structure was done by Pymol. FAD and THF are in yellow color. The possible connection of FAD and THF sites is shown by the yellow line. 


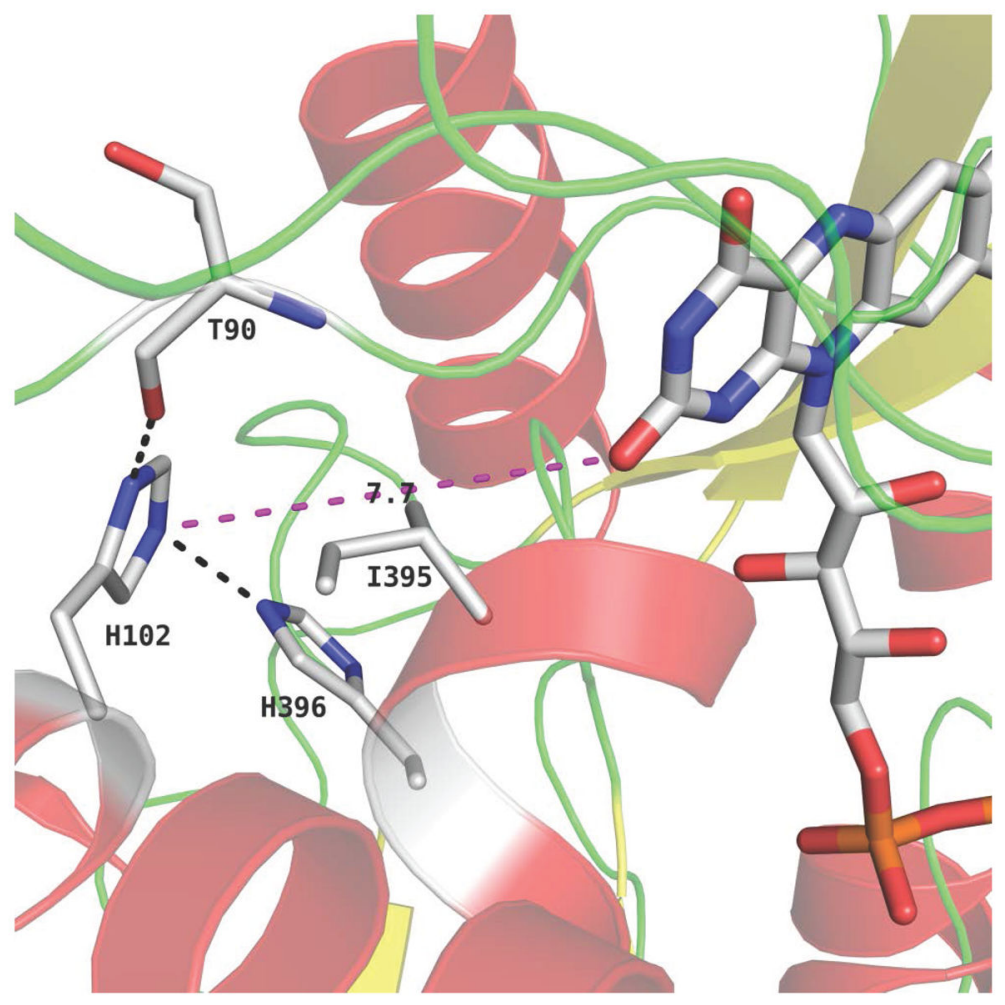

Figure 4. Modeling the position of human His109 in the crystal structure

The interactions of the His102 in rat DMGDH which corresponds to His109 in the sequence of human DMGDH in the crystal structure. 


\section{Table 1}

Data Collection, Phasing and Refinement Statistics.

\begin{tabular}{|c|c|c|c|}
\hline & DMGDHvar2-folate & mDMGDH - folate & mDMGDH \\
\hline Wavelength $(\AA)$ & 0.9787 & 0.9787 & 0.9787 \\
\hline Resolution ( $\mathrm{A})$ & 1.85 & 2.25 & 2.32 \\
\hline Temperature (K) & 100 & 100 & 100 \\
\hline Space group & $P 2_{1}$ & $P 2_{1} 2_{1} 2_{l}$ & $P 2_{1} 2_{l} 2_{l}$ \\
\hline \multicolumn{4}{|l|}{ Cell dimensions } \\
\hline$a(\AA)$ & 84.18 & 84.93 & 85.21 \\
\hline$b(\AA)$ & 107.22 & 130.66 & 131.44 \\
\hline$c(\AA)$ & 114.47 & 171.86 & 171.92 \\
\hline$\beta\left(^{\circ}\right)$ & 92.2 & & \\
\hline Number of molecules per asymmetric unit & 2 & 2 & 2 \\
\hline No. of unique reflections & 167803 & 87235 & 75192 \\
\hline $\mathrm{R}_{p i m}^{1,2}(\%)$ & $3.7(39.2)$ & $6.1(29.7)$ & $7.6(29.8)$ \\
\hline Completeness (\%) & $96.7(90.2)$ & $97.1(90.6)$ & $91.1(85.5)$ \\
\hline Redundancies & $5.7(4.3)$ & $6.7(5.6)$ & $5.6(6.0)$ \\
\hline$I / \sigma(I)$ & $22.2(1.5)$ & $11.7(1.9)$ & $13.7(2.3)$ \\
\hline \multicolumn{4}{|l|}{ Refinement statistics } \\
\hline Resolution range $(\AA)$ & $48.19-1.85$ & $40-2.26$ & $40-2.32$ \\
\hline No. of reflections used in refinement & 163455 & 85428 & 73606 \\
\hline$\sigma$ cutoff used in refinement & none & none & none \\
\hline$R / R_{\text {free }}{ }^{3}(\%)$ & $20.65 / 23.04$ & $24.08 / 26.74$ & $25.30 / 29.48$ \\
\hline \multicolumn{4}{|l|}{ Number of refined atoms } \\
\hline Protein & 13085 & 12952 & 12952 \\
\hline Heterogen atoms & 178 & 170 & 106 \\
\hline Water & 721 & 236 & 104 \\
\hline \multicolumn{4}{|l|}{ Average B-factors $\left(\AA^{2}\right)$} \\
\hline Protein & 35.8 & 38.3 & 31.0 \\
\hline Water & 30.5 & 15.2 & 16.8 \\
\hline FAD & 25.1 & 16.8 & 21.3 \\
\hline THG & 50.8 & 30.9 & - \\
\hline \multicolumn{4}{|l|}{ R.m.s. deviations } \\
\hline Bonds $(\AA)$ & 0.008 & 0.007 & 0.011 \\
\hline Angles $\left({ }^{\circ}\right)$ & 1.288 & 1.168 & 1.444 \\
\hline \multicolumn{4}{|l|}{ Ramachandran plot (\%) } \\
\hline Favored & 97.32 & 97.17 & 96.44 \\
\hline Outliers & 0.12 & 0.12 & 0.12 \\
\hline
\end{tabular}


$R_{\text {pim }}=\Sigma_{h k l}(1 /(n-1))^{1 / 2} \Sigma_{i} \mid I_{h k l, i}-\left\langle I_{h k l}>\right| \Sigma_{h k l} \Sigma_{i} I_{h k l, i}$, where $I_{h k l, i}$ is the scaled intensity of the $i^{t h}$ measurement of reflection h, k, 1 , $\left\langle I_{h k l}\right\rangle$ is the average intensity for that reflection, and $n$ is the redundancy.

${ }^{3} R=\Sigma|| F_{O}|-| F_{C} \| / \Sigma\left|F_{O}\right|$, where $F_{O}$ and $F_{C}$ are the observed and calculated structure factors amplitudes. $R$ free is calculated using $\sim 2.0 \%$ of reflections omitted from the refinement. The same $R_{\text {free }}$ set was used in case of mDMGDH-folate and mDMGDH structures because of isomorphous character of data sets. 